\title{
Ring-shaped exact Hopf solitons
}

\author{
C. Adam ${ }^{1}$ and J. Sánchez-Guillén ${ }^{2}$ \\ ${ }^{1}$ Wolfgang Pauli Institut c/o Institut für Mathematik, \\ Universität Wien, \\ 1090 Vienna, Austria \\ 2 Departamento de Física de Partículas, \\ Facultad de Física \\ Universidad de Santiago \\ E-15706 Santiago de Compostela, Spain
}

\begin{abstract}
The existence of ring-like structures in exact hopfion solutions is shown.
\end{abstract}




\section{Introduction}

Extended solutions are of central importance in different applications of modern field theory, from high energy to condensed matter physics. Their relevance is parallel to the difficulty in the analysis of the nonlinear theories which encode them and the scarce exact results in higher dimensions. To circumvent one of the main problems, the scaling instablity for scalar fields beyond one spatial dimension found by Skyrme [1] and formalized by Derrick [2], one obvious possibility is nonpolynomial lagrangians, which are not unfamiliar. Among those attempts the work by Deser, Duff and Isham [3], where the simplest choice of just the power required to balance the scaling is analyzed, is of special interest. As the authors discuss, such models have, of course, no free field expansion around a trivial vacuum. But they can have a semiclassical formulation around nontrivial solutions and eventually a small time dependent perturbation of the static solutions. One solution was found and the importance of global transformations was emphasized. This model was further extended by Nicole [4] and put in a more general framework by Kundu [5]. It was rediscovered independently in a series of papers [6] in the context of a new proposal for a generalized integrability [7], finding infinitely many analytic solutions with general Hopf indices. This was an unsolved problem, which is important for soliton physics and because of the many applications of those maps, combining topology and geometry. In their last paper [8], a new feature of the Hopfion solutions was discovered, namely, a line singularity in an infinitely thin tube along the z-axis from a special current with a non-conserved charge, provided by the geometric method.

In this Letter we extend the analysis to find that there is in fact another solution of the ring type, which is interesting, as ring structures are typical in higher dimensional soliton analysis, both in numerical [9] and analytical approximations [10]. In fact, these currents and solutions should be relevant for the analytical study of soliton scattering. We pay also special attention to the symmetries, including global aspects of the solution, as many aspects of the model are generic to other local formulations of topological degrees of freedom. Time dependence analysis is also a natural possiblity in the the Generalized Zero Curvature approach, as it preserves Lorentz covariance. 


\section{The ring-like solutions}

As shown in [8], the only models involving the antisymmetric tensor in the complex field $u$

$$
h_{\mu \nu}=-i\left(\partial_{\mu} u \partial_{\nu} u^{*}-\partial_{\nu} u \partial_{\mu} u^{*}\right)
$$

which satisfy without constraints the integrability criterion of the geometric approach of [7] (i.e. infinitely many conserved currents) and which can be derived from an action principle, are those where the lagrangian is a functional of $h^{2} / f^{2}$, where $f$ is a real function of $u$ and $u^{*}$. If one further asks for scaling invariance to allow for stable static solutions, we are lead to the class of models given by the Lagrangian density

$$
\mathcal{L} \equiv\left(\frac{h_{\mu \nu}^{2}}{2 f^{2}}\right)^{\frac{3}{4}}
$$

which generalizes and explains choices and solutions found before [3] - [6].

We assume from now on that $f$ only depends on $u u^{*}$. If $\lim _{|\vec{x}| \rightarrow \infty} u(\vec{x})=u_{0}=$ const is assumed, then the domain space $I R^{3}$ has the topology of the three-sphere $S^{3}$. If, in addition, the target space may be identified with the two-sphere $S^{2}$ via stereographic projection, then $u$ may be interpreted as a map $S^{3} \rightarrow S^{2}$, which is characterized by an integer winding number (the Hopf index). In this case there exists an infinite number of static, soliton-like solutions to the equations of motion of the model, and these all have integer Hopf index. These solutions were first found in [6] and they are obtained by inserting into the static equations of motion

$$
-h_{i j} \partial^{j} u \partial^{i} h^{2}+4 h^{2} \partial^{i} h_{i j} \partial^{j} u+i\left(h^{2}\right)^{2} \partial_{u^{*}} f=0
$$

(where $h^{2} \equiv h_{i j} h^{i j}$ ) the product ansatz

$$
u(\eta, \xi, \varphi) \equiv R(\eta) e^{i(m \xi+n \varphi)}
$$

in toroidal coordinates

$$
\begin{aligned}
& x=q^{-1} \sinh \eta \cos \varphi, y=q^{-1} \sinh \eta \sin \varphi \\
& z=q^{-1} \sin \xi \quad ; q=\cosh \eta-\cos \xi
\end{aligned}
$$

As explained elegantly in [8], the Ansatz (4) follows from the conformal symmetry of the equations of motion. If one assumes that $f$ is a function of $T \equiv R^{2} \equiv u u^{*}$ only, $\partial_{u^{*}} f$ simplifies to $\partial_{u^{*}} f=f_{, T} u$. With the Ansatz (4), this results in an ordinary differential equation for $R(\eta)$, which may be conveniently expressed in terms of $T \equiv R^{2} \equiv u u^{*}$ as

$$
\left(\ln \frac{T_{, \eta}}{f}\right)_{, \eta}=\frac{\cosh \eta}{\sinh \eta} \frac{n^{2}-2 m^{2} \sinh ^{2} \eta}{n^{2}+m^{2} \sinh ^{2} \eta}
$$


and $X_{, \eta}$ denotes derivative of $X$ w.r.t. $\eta$. Further, we assume $m^{2}>n^{2}$ in the sequel. A first integral may be found easily,

$$
\frac{T_{, \eta}}{f}=k_{1} \frac{\sinh \eta}{\left(n^{2}+m^{2} \sinh ^{2} \eta\right)^{3 / 2}}
$$

(here $k_{1}$ is a constant of integration), whereas for a further integration the explicit form of the function $f(T)$ is needed.

In the end, we shall choose $f=(1+T)^{2}$, because we are refering to the solutions of Babelon and Ferreira [8], but let us briefly mention a class of functions $f(T)$ that leads to a target space with the topology of the two-sphere and, therefore, to genuine Hopf solitons, which can have useful applications. For $f=(1+T)^{2}$, the expression $h_{i j} / f$ in the Lagrangian density (2) is, in fact, just the pull-back under the map $u$ of the area two-form

$$
d \Omega=-i \frac{d z d z^{*}}{\left(1+z z^{*}\right)^{2}}
$$

on the two-sphere. A pull-back of this two-form under maps $S^{2} \rightarrow S^{2}$ will lead to further acceptable area two-forms (i.e. area two-forms respecting the topology of the target space). If we want to maintain the simple dependence $f=f(T)$, then a class of allowed maps is

$$
\phi: z \rightarrow \sqrt{g\left(z z^{*}\right)} e^{i l \arg (z)} .
$$

These are indeed maps $S^{2} \rightarrow S^{2}$ provided that $g(0)=0$ and $g(\infty)=\infty$. Further, $l$ must be an integer. The pullback of the area two-form (8) is

$$
\phi_{*}(d \Omega)=-i \frac{d z d z^{*} l g^{\prime}}{(1+g)^{2}}
$$

therefore any function $f$ of the type

$$
f(T)=\frac{(1+g(T))^{2}}{\lg _{, T}}
$$

leads to a theory (2) with genuine Hopf solitons.

In the sequel we restrict to the simplest case $f=(1+T)^{2}$ (the area two-form on the two-sphere). Then the first integral (7) may be easily integrated to yield

$$
\frac{1}{1+T}=\frac{k_{1}}{\left(m^{2}-n^{2}\right)} \frac{\cosh \eta}{\left(n^{2}+m^{2} \sinh ^{2} \eta\right)^{1 / 2}}+k_{2}
$$

where $k_{1}$ and $k_{2}$ are two constants of integration. These constants have to be fixed by imposing some boundary conditions on the field $u$. For this purpose let us introduce the unit vector $\vec{n}$ related to $u$ via stereographic projection

$$
\vec{n}=\frac{1}{1+|u|^{2}}\left(u+u^{*},-i\left(u-u^{*}\right),|u|^{2}-1\right) ; \quad u=\frac{n_{1}+i n_{2}}{1-n_{3}} .
$$


If $u$ is supposed to be a true Hopf map, then the number of allowed boundary conditions is, in fact, very restricted. The point is that a true Hopf map should cover the whole target $S^{2}$, including the north pole $(\vec{n}=(0,0,1)$, or $T=\infty)$ and the south pole $(\vec{n}=(0,0,-1)$, or $T=0)$. Therefore, the boundary conditions should be chosen such that $T$ varies between $T=0$ and $T=\infty$. Further, the pre-images of the north pole and the south pole should be one-dimensional lines in $I R^{3}$. However, the only values of $\eta$ which define one-dimensional lines (instead of two-dimensional tori, as is the general case), are $\eta=0$, which defines the $z$ axis (together with spatial infinity), and $\eta=\infty$, which defines the circle

$$
C=\left\{\vec{x} \in \mathbb{R}^{3}: z=0 \wedge r^{2}=1\right\} .
$$

Therefore, there are two possible choices for the boundary conditions, namely $T(\eta=$ $0)=0, T(\eta=\infty)=\infty$, or $T(\eta=0)=\infty, T(\eta=\infty)=0$.

In [8] the second option was chosen (which we call $T^{(2)}$ for convenience),

$$
T^{(2)}=\frac{\cosh \eta-\sqrt{n^{2} / m^{2}+\sinh ^{2} \eta}}{\sqrt{1+m^{2} / n^{2} \sinh ^{2} \eta}-\cosh \eta}, T^{(2)}(\eta=0)=\infty, T^{(2)}(\eta=\infty)=0 .
$$

Let us now investigate our first option, for which we get

$$
T^{(1)}=\left(T^{(2)}\right)^{-1}, T^{(1)}(\eta=0)=0, T^{(1)}(\eta=\infty)=\infty .
$$

Now one could simply identify the solitons with the position of their singular value $|u|=\infty$, then the solitons of type $T^{(2)}$ would be identified with the straight line $x=0, y=0$ (the $z$ axis), and the solitons of type $T^{(1)}$ would be ascribed to the circle $C$. However, this identification is in principle quite arbitrary, and requires a physical motivation.

In [8] it was shown that the solitions of type $T^{(2)}$ can indeed be identified with the $\mathrm{z}$ axis in a well-defined manner. More precisely, there exists a conserved current $J_{\mu}$ which has the property that for solitons of type $T^{(2)}$ it is singular along the $z$ axis. Moreover, a constant flow of this current is emerging from the singular line (the $z$ axis). Here we want to investigate the equivalent problem for solitons of type $T^{(1)}$, which is relevant as rings are common in higher dimensional solitons [9], [10].

So let us briefly review and further develop some results of [8] on the abovementioned conserved current, with some more details required for our purposes. There exists (among other symmetries) a symmetry of the action under a transformation which is a combination of a dilatation on three-dimensional domain space plus a specific transformation on target space. The infinitesimal version of this symmetry transformation is given in [8], but it is, in fact, not difficult to obtain the transformation for 
finite transformation parameter. Under a dilatation transformation $x \rightarrow \Lambda^{3} x \equiv e^{3 \lambda} x$ (the power three of the dilatation parameter is chosen for later convenience) the action of the theory scales like

$$
S \rightarrow \Lambda^{-3} S
$$

therefore the theory is invariant if the dilatation is combined with a transformation of the target space variable, $u \rightarrow v(u, \bar{u})$, such that

$$
\frac{d u d \bar{u}}{(1+\bar{u} u)^{2}} \rightarrow \frac{d v d \bar{v}}{(1+\bar{v} v)^{2}}=\Lambda^{2} \frac{d u d \bar{u}}{(1+\bar{u} u)^{2}}
$$

If we introduce the real coordinates on target space $u=T^{1 / 2} e^{i \phi}$ (angle and radius squared on the Euclidean plane) and assume that $v=(\tilde{T})^{1 / 2}(T) e^{i \phi}$ (i.e. $u$ and $v$ have the same argument, and the modulus of $v$ is a function of the modulus $T$ only) then we get the equation

$$
\frac{\tilde{T}^{\prime}(T) d T d \phi}{(1+\tilde{T})^{2}}=\Lambda^{2} \frac{d T d \phi}{(1+T)^{2}}
$$

or

$$
\frac{\tilde{T}^{\prime}}{(1+\tilde{T})^{2}}=\frac{\Lambda^{2}}{(1+T)^{2}}
$$

with the solution

$$
\frac{1}{1+\tilde{T}}=\frac{\Lambda^{2}}{1+T}+c
$$

where $c$ is a constant of integration. If we require the boundary condition $\tilde{T}(0)=0$ then we get

$$
\tilde{T}=\frac{\Lambda^{2} T}{\Lambda^{2}+(1+T)\left(1-\Lambda^{2}\right)}
$$

or

$$
v=\frac{\Lambda u}{\left[\Lambda^{2}+(1+\bar{u} u)\left(1-\Lambda^{2}\right)\right]^{1 / 2}} .
$$

This $v$ indeed fulfills Eq. (18) as may be checked easily. In addition, it reduces to the transformation of Babelon and Ferreira [8] for infinitesimal $\lambda$ (i.e. $\Lambda=1+\lambda$ ). Therefore Eq. (23) is the required symmetry transformation on target space. ${ }^{1}$

The conserved Noether current related to this symmetry transformation is

$$
J_{\mu}=x^{\nu} \Theta_{\mu \nu}+j_{\mu}
$$

where $\Theta_{\mu \nu}$ is the canonical energy-momentum tensor of the theory, and $j_{\mu}$ is

$$
j_{\mu}=-i\left(\frac{h^{2}}{2(1+T)^{4}}\right)^{-1 / 4} \frac{1}{2(1+T)^{3}} h_{\mu \nu}\left(u \partial^{\nu} u^{*}-u^{*} \partial^{\nu} u\right)
$$

\footnotetext{
${ }^{1}$ Observe that this transformation has the funny property that it is well-defined only for $\Lambda^{2} \leq 1$, i.e., for scaling transformations which shrink distances .
} 
Here, the first term of $J_{\mu}$ (containing the energy-momentum tensor) is due to the space dilatation, whereas the second term, $j_{\mu}$, is due to the specific target space transformation (23). The current obeys the conservation equation $\partial^{\mu} J_{\mu}=0$. For static configurations $u(\vec{x})$ this conservation equation may be used to derive the relation

$$
E \equiv \partial_{t} \int d^{3} x J^{0}=\int_{\Sigma} d \vec{f} \cdot \vec{J}
$$

where $E$ is the static energy of the static configuration,

$$
E=4 \pi^{2} \sqrt{|m||n|(|m|+|n|)}
$$

and the integral in the r.h.s. of (26) is an integral over surfaces $\Sigma$ which surround the singularities of the current $\vec{J}$. It turns out that the first term of the current for static fields, $x^{j} \Theta_{i j}$, is regular everywhere and may, therefore, be ignored in the surface integral of (26) provided that the integration surfaces are chosen such that the enclosed volume is infinitesimal. For the current $\vec{j}$ we find after some calculation

$$
\vec{j}=\left(\left|\frac{T_{, \eta}}{(1+T)^{2}}\right|\right)^{1 / 2} \frac{T}{1+T}\left(\frac{n^{2}}{\sinh ^{2} \eta}+m^{2}\right)^{3 / 4}(\cosh \eta-\cos \xi)^{2} \vec{e}_{\eta}
$$

where $\vec{e}_{\eta}=(\cosh \eta-\cos \xi)^{-1} \nabla \eta$ is a vector of unit length which is perpendicular to the surfaces of constant $\eta$ (tori). Observe that $\vec{e}_{\eta}$ is pointing into the interior of the tori, because $\eta$ is growing in this direction. We may use the first integral of the equations of motion, (7), for the first factor on the r.h.s. containing $T_{, \eta}$, and find

$$
\vec{j}=\sqrt{|m||n|(|m|+|n|)} \frac{T}{1+T} \frac{(\cosh \eta-\cos \xi)^{2}}{\sinh \eta} \vec{e}_{\eta} .
$$

If we ignore the factor $T /(1+T)$ for the moment, then we see that the remaining expression is singular both for $\eta=0$ (along the $z$ axis) and for $\eta=\infty$ (along the circle $C)$. Depending on whether we choose $T^{(1)}$ or $T^{(2)}$ for $T$, one of the two singularities gets cancelled, whereas the other remains. For $T=T^{(2)}$ (the case which was studied in [8]), the singularity along the $z$ axis remains. In this case we choose a very large torus $\eta<<1$ as integration surface. For the regular terms the limit $\eta \rightarrow 0$ may be performed, such that the integration is extended to the whole space. For the singular $\vec{j}$ the surface integral should be performed for a finite $\eta$ and the limit $\eta \rightarrow 0$ should be taken afterwards. The surface element on the torus surface $\eta=$ const is

$$
d \vec{f}=\vec{e}_{\eta} \frac{\sinh \eta}{(\cosh \eta-\cos \xi)^{2}} d \varphi d \xi
$$

therefore the surface integral is

$$
\begin{aligned}
\int_{\eta=\mathrm{const}} \vec{j} \cdot d \vec{f} & =\sqrt{|m||n|(|m|+|n|)} \frac{T}{1+T} \int d \varphi d \xi \\
& =4 \pi^{2} \sqrt{|m||n|(|m|+|n|)} \frac{T}{1+T}
\end{aligned}
$$


For $T=T^{(2)}$ this should be evaluated in the limit $\eta \rightarrow 0$ for which $T^{(2)} /\left(1+T^{(2)}\right)$ is equal to one. Therefore the total flux emerging from the singular line (the $z$ axis) is

$$
\text { flux }=4 \pi^{2} \sqrt{|m||n|(|m|+|n|)}=E
$$

where $E$ is the static energy (27).

On the other hand, for $T=T^{(1)}$ the singularity is located at the circle $C$, therefore a tiny torus (large $\eta$ ) should be excluded from the integration region. So we take the limit $\eta \rightarrow \infty$ now in the surface integral (31). But $T^{(1)} /\left(1+T^{(1)}\right)$ is equal to one in this limit, so we find again for the flux the same previous expression (32) .

Therefore, for solutions of the type $T^{(1)}$ the singular line is the circle $C$ and a non-zero flux of total amount given in (32) emerges from this singular circle. As a consequence, the solutions of type $T^{(1)}$ are characterized by a ring-like structure, where the ring is located at the position of the circle $C$, which, as said, is relevant for their physics and specially for the scattering.

\section{Discussion}

So we indeed found that in addition to the solutions of type $T^{(2)}$ originally obtained by Aratyn, Ferreira and Zimerman (AFZ) in [6], which are characterized by a straight line of singular flux according to Babelon and Ferreira, there exist solutions where the singular flux is located along the circle $C$, forming thereby a ring-like structure. In hindsight, this result is not so surprising, and the ring-like structure is, in fact, the generic case. To see this, let us invoke a further symmetry of the model, namely constant rotations of the target space $S^{2}$. In domain space $I R^{3}$ such a rotation rotates different level curves (i.e., curves of constant $u=u_{0}$ for different values of $u_{0}$ ) into each other, because these level curves are the pre-images of points of the target $S^{2}$ under the map $u$. Generically, these level curves are circles, with the only exception of the $z$ axis. Therefore, any rotation on target space which moves the north pole and the south pole will transform a solution of type $T^{(2)}$ into a new solution where the line of singular flux is located along a circle.

On the target space coordinate $u$ such rotations are represented by modular transformations $u \rightarrow(a+b u) /(c+d u)$, where $a d-b c=1$. A general modular transformation is, however, not compatible with the simple Ansatz (4) which was used by AFZ to find solutions. The only non-trivial modular transformation compatible with the ansatz (4) is the inversion map $u \rightarrow(1 / u)$. And indeed, the composition of the inversion map with the map $(m, n) \rightarrow(-m,-n)$ (which again maps a solution to another solution 
with the same energy) precisely maps the AFZ solutions $T^{(2)}$ to the solutions of type $T^{(1)}$ discussed in this paper.

Besides their interest for the scattering, the results can also be useful - given the generic features of the theory considered - for other higher dimensional models on the sphere and/or using similar scaling arguments, like Skyrme theory and its restriction to the $S U(2) / U(1)$ coset, proposed by Faddeev as an effective theory of QCD at long distances [11].

Acknowledgment: CA acknowledges support from the Austrian START award project FWF-Y-137-TEC of N.J. Mauser and J S-G support from the Spanish MCyT and FEDER projects FPA2002-01161 and BFM2002-03881 and discussions with L.A. Ferreira.

\section{References}

[1] T.H.R. Skyrme, A nonlinear field theory, Proc. Roy. Soc. Lon. 260 (1961) 127.

[2] G.M. Derrick, Comments on non-linear wave equations as models for elementary particles, J. Math. Phys. 5 (1964) 1252.

[3] S. Deser, M.J. Duff, C.J. Isham, Finite-energy static solutions to chiral models in three space dimensions, Nucl. Phys. B114 (1976) 29.

[4] D.A. Nicole, Solitons with non-vanishing Hopf index, J. Phys. G4 (1978) 1363.

[5] A. Kundu, Construction of nonlinear $\sigma$-models in higher space dimensions, Annals of Physics 139 (1982) 36.

[6] H. Aratyn, L.A. Ferreira and A. Zimerman, Toroidal solitons in $3+1$ dimensional integrable theories, Phys. Lett. 456B (1999) 162. Exact static soliton solutions of $3+1$ dimensional integrable theory with nonzero Hopf numbers, Phys. Rev. Lett. 83 (1999) 1723.

[7] Orlando Alvarez, L.A. Ferreira and J. Sánchez-Guillén, A new approach to integrable theories in any dimension, Nucl. Phys. B529 (1998) 689.

[8] O. Babelon and L.A. Ferreira, Integrability and conformal symmetry in higher dimensions: a model with exact Hopfion solutions, JHEP 0211 (2002) 020. 
[9] P.M. Sutcliffe, Nontrivial soliton scattering in an integrable chiral model in $(2+1)$ dimensions, J. Math. Phys. 33 (1992) 2269

[10] R.S. Ward, Slowly moving lumps in the $C P^{1}$ model in $(2+1)$ dimensions, Phys. Lett. 158B (1985) 424. The Interacion of two Hopf solitons, Phys. Lett. 473B (2000) 291.

[11] L. Faddeev, Einstein and several contemporary tendencies in the Field Theory of Elementary particles, in Relativity, Quanta and Cosmology vol1. M. Pantaleo and F. De Finis (eds.) Johnson Reprint, 1979 and in 40 Years in Mathematical Physics, (World Scientific, 1995). L. Faddeev and A. Niemi, Nature 387 (1997) 58. L. Faddeev and A.I. Niemi, Phys. Lett. 525B (2001) 195. 